\title{
Intentional binding coincides with explicit sense of agency
}

Shu Imaizumi ${ }^{\mathrm{a}, \mathrm{b}^{*}}$, Yoshihiko Tanno ${ }^{\mathrm{a}}$

${ }^{a}$ Graduate School of Arts and Sciences, The University of Tokyo, 3-8-1 Komaba, Meguro, Tokyo 153-8902, Japan

${ }^{\mathrm{b}}$ Japan Society for the Promotion of Science, 5-3-1 Kojimachi, Chiyoda, Tokyo 102-0083, Japan

"Corresponding author: Shu Imaizumi. Graduate School of Arts and Sciences, The University of Tokyo, 3-8-1 Komaba, Meguro, Tokyo 153-8902, Japan. E-mail: shuimaizumi@gmail.com

Abstract: 150 words

Keywords: 6

Main text: 12,517 words

Figures: 6

Table: 1

References: 89

Note: Color should be used for all figures in print.

\begin{abstract}
Sense of agency, a feeling of generating actions and events by oneself, stems from action-outcome congruence. An implicit marker of sense of agency is intentional binding, which is compression of subjective temporal interval between action and outcome. We investigated relationships between intentional binding and explicit sense of agency. Participants pressed a key triggering auditory (Experiment 1) or visual outcome (Experiment 2) that occurred after variable delays. In each trial, participants rated their agency over the outcome and estimated the keypress-outcome temporal interval. Results showed that delays decreased agency ratings and intentional binding. There was inter-individual correlation between sensitivities to outcome delay (i.e., regression slope) of agency rating and intentional binding in the auditory but not visual domain. Importantly, we found intra-individual correlations between agency rating and intentional binding on a trial-by-trial basis in both outcome modalities. These results suggest that intentional binding coincides with explicit sense of agency.
\end{abstract}

\section{Keywords}

self awareness; action; sensorimotor; sense of agency; time perception; intra-individual variation 


\section{Introduction}

\subsection{Sense of agency}

We, as voluntary agents, normally experience a sense of agency. This refers to a feeling and/or awareness of generating one's own actions (Gallagher, 2000), and through them, events in the external world (Haggard, 2017). We may routinely feel that, for example, "my arm is being moved by me" and "I turned on that TV." Sense of agency seems to be an innate, ubiquitous sensation, but stems from the sensorimotor system of the brain and body, while being modulated by social factors (Caspar, Christensen, Cleeremans, \& Haggard, 2016; Obhi \& Hall, 2011; Yoshie \& Haggard, 2013).

One key factor for generating sense of agency is active movements involving motor commands. For instance, it has been empirically demonstrated that active, but not passive, movement is likely to generate sense of agency over sensory outcomes (Engbert, Wohlschlager, \& Haggard, 2008; Haggard, Clark, \& Kalogeras, 2002). Behind voluntary active movements, internal forward models in the sensorimotor system predict sensory feedback (e.g., auditory, visual, and somatosensory) based on efference copies of preceding motor commands for planned movements (Wolpert, Ghahramani, \& Jordan, 1995). Then, a "comparator" implemented in the forward model compares the actual sensory feedbacks against the predicted feedback (Frith, Blakemore, \& Wolpert, 2000); if spatially and temporally matched, sense of agency will be generated. Alternatively, if spatio-temporally unmatched, the prediction error disrupts sense of agency, and consequently the violated prediction and relevant motor command will be corrected. For instance, delayed auditory (Asai \& Tanno, 2008; Sato \& Yasuda, 2005) and visual (Farrer, Valentin, \& Hupe, 2013; Franck et al., 2001) feedback from voluntary action (e.g., keypress, joystick control), which does not spatio-temporally match the prediction, can decrease perceived sense of agency. Aside from these retrospective "comparator" mechanisms, sense of agency can also be prospectively modified by, for example, a priori likelihood of sensory outcome (Moore \& Haggard, 2008), fluency of action selection (Chambon \& Haggard, 2012), and range of action options (Barlas \& Obhi, 2013) before action execution.

\subsection{Explicit and implicit measures of sense of agency}

There is one view that sense of agency consists of explicit and implicit levels (Synofzik, Vosgerau, \& Newen, 2008). The explicit level is characterized by reflective, inferential, and belief-like aspects, while the implicit level is characterized by its pre-reflective aspects. Accordingly, studies on sense of agency have employed explicit (direct) and implicit (indirect) measures (David, Newen, \& Vogeley, 2008). For explicit measures, binary judgment and Likert-scale ratings have been used. For example, studies have employed binary responses on whether cursor movement on a monitor is attributable either to oneself or to another individual (Franck et al., 2001), as well as a Likert rating scale to 
ascertain the extent of agreement with a statement such as "I caused the sensory outcome" (Sato \& Yasuda, 2005). When spatial and temporal biases are inserted into sensory feedback of participant's movements, explicit measures of agency (i.e., rating scores and proportion of "self" responses) decrease as a function of the degree of spatio-temporal bias. Importantly, explicit measures of agency and bias-detection performance show distinct psychometric functions, suggesting that explicit measure does not merely reflect the detection of the experimental manipulations per se, but indeed reflects altered sense of agency (Asai \& Tanno, 2007, 2013).

Alternatively, using implicit measures, studies have shown reductions in perceptual intensity and neural responses for self-initiated sensory events (Blakemore, Wolpert, \& Frith, 2000), and compression of the subjective interval between an action and its outcome observed in active, but not passive, movement (Haggard et al., 2002). The latter finding, called "intentional binding," has been used extensively in studies of sense of agency (Haggard, 2017; Moore \& Obhi, 2012). Intentional binding can be measured mainly in two ways: a clock reading paradigm (Libet, Gleason, Wright, \& Pearl, 1983), where participants report the clock-hand position at the time when they pressed the key or heard a tone following the keypress (Haggard et al., 2002); or an interval estimation paradigm where participants verbally estimate the temporal interval between an action and its outcome (Engbert, Wohlschlager, Thomas, \& Haggard, 2007). Intentional binding, as well as explicit judgment of agency, can be reduced by temporal discrepancy between an action and its outcome in healthy individuals (Haggard et al., 2002; Ruess, Thomaschke, \& Kiesel, 2018). From a psychiatric perspective, both implicit and explicit measures of the sense of agency can also reflect the abnormal sense of agency in schizophrenia. Intentional binding can be abnormally enhanced in people with schizophrenia (Haggard, Martin, Taylor-Clarke, Jeannerod, \& Franck, 2003; Moore et al., 2011; Voss et al., 2010). They are also likely to experience abnormal explicit sense of agency, namely, the misattribution of externally initiated sensory events to the self (Hur, Kwon, Lee, \& Park, 2014) and the misattribution of self-initiated action-outcome to the other (Waters, Woodward, Allen, Aleman, \& Sommer, 2012) due to a dysfunction of motor prediction and a deterioration of self-other discriminability (Asai, 2016). The above findings from healthy people and patients suggest that explicit and implicit aspects of a sense of agency can be related to each other, although they may originate through different pathways (Synofzik et al., 2008; Synofzik, Vosgerau, \& Voss, 2013).

\subsection{Relationship between agency judgment and intentional binding}

The relationship between sense of agency and time perception is not limited to intentional binding. Self-initiated dot movements with an orientation corresponding to prediction are perceived to move slower than externally initiated moving dots (Dewey \& Carr, 2013), suggesting that sense of agency 
itself may modulate time perception during active movements. Our previous study has shown that agency and duration judgments for concurrent video feedback of manual action were similarly modulated by feedback delays: actions with and without sense of agency are perceived to have a longer or shorter duration than baseline, respectively (Imaizumi \& Asai, 2017). Therefore, it can be hypothesized that intentional binding is not simply a marker of sense of agency, but also a behavioral signature of time perception that is modulated by sense of agency. Indeed, intentional binding can entail dynamic modulation of perceptual temporal resolution and/or the internal clock (Wenke \& Haggard, 2009, but see also Fereday \& Buehner, 2017).

One important question is whether intentional binding (i.e., implicit sense of agency) and explicit sense of agency correlate. In fact, brain imaging and stimulation literature suggests that these two aspects of agency may be associated with different brain areas. Intentional binding can be modulated by the frontal regions, especially the pre-supplementary motor area (pre-SMA) (Moore, Ruge, Wenke, Rothwell, \& Haggard, 2010) and the left dorso-lateral prefrontal cortex (Khalighinejad, Di Costa, \& Haggard, 2016). In contrast, explicit sense of agency can be modulated by parietal regions, such as the right angular gyrus (Farrer, Frey, et al., 2008; Farrer \& Frith, 2002) and the right temporoparietal junction (Hughes, 2018). However, meta-analytic study suggests that not only temporoparietal junction but also pre-SMA is associated with explicit sense of agency (Sperduti, Delaveau, Fossati, \& Nadel, 2011). Indeed, pre-SMA can be involved in the monitoring and controlling of action (Nachev, Rees, Parton, Kennard, \& Husain, 2005; Nachev, Wydell, O'Neill, Husain, \& Kennard, 2007), which determine explicit sense of agency through the comparator and prospective mechanisms. Furthermore, fluency of action, which is also a determinant of the sense of agency, can be associated with functional connectivity between the dorso-lateral prefrontal cortex and the angular gyrus in parietal cortex (Chambon, Wenke, Fleming, Prinz, \& Haggard, 2013). Taken together, although intentional binding and explicit sense of agency may distinctively stem from frontal and parietal regions, respectively, these two aspects of agency might indeed be coupled at a neural level and modulated by shared mechanisms (i.e., the comparator process and the action monitoring).

If our argument above is plausible, there would be a behavioral correlation between intentional binding and explicit sense of agency. Although some behavioral studies (Braun, Thorne, Hildebrandt, \& Debener, 2014; Dewey \& Knoblich, 2014; Saito, Takahata, Murai, \& Takahashi, 2015) have reported that this is not the case, the methodologies used have room for improvement. Braun et al. (2014) measured explicit sense of agency over a fake hand moving in correspondence with the participant's own hand (Kalckert \& Ehrsson, 2012), and measured intentional binding by estimating the temporal interval between the rubber hand's keypress and the subsequent tone, in separate 
experimental blocks. Saito et al. (2015) measured explicit agency over movement of the virtual hand induced by the participant's joystick movement, but measured implicit agency (i.e., intentional binding) using a quite different task, specifically, the estimation of subjective timing of a keypress and a single tone. Given that measurements were made in separate blocks and in different experimental situations, these studies may have failed to purely compare and relate agency judgment with intentional binding. Overcoming these issues, Dewey and Knoblich (2014) measured intentional binding using keypress-tone interval estimation, and measured subjective ratings of the sense of agency over the tone in an identical experimental setting. However, as Dewey and Knoblich administered the agency-rating trial once every 45 interval-estimation trials, the lack of correlation reported between the two measures might be due to an artefact of unbalanced experimental design. Other studies have shown a correlation between agency judgment and intentional binding, while overcoming the above methodological issues. Ebert and Wegner (2010) used images of natural objects moving forward/backward (in)consistently with participant's joystick movements. In each trial, they measured agency rating over the visual stimuli and subjective temporal interval between the joystick movement and stimulus movement. As a result, the differential scores of agency ratings between consistent and inconsistent conditions were positively correlated with those of estimated intervals. A recent study (Pyasik, Burin, \& Pia, 2018) further demonstrated the correlation between agency ratings and intentional binding by using Libet's clock task and ratings of agency over the tone in each single trial. Nevertheless, it should also be kept in mind that the correlation between explicit and implicit measures of agency disappeared when they were separately measured in different blocks (Ebert \& Wegner, 2010), implying that the attention to awareness of one's agency and perceived passage of time has a potential role for the relationship between explicit and implicit aspects of agency.

In Synofzik et al. (2008)'s model, the implicit (i.e., pre-reflective) level of a sense of agency originates from sensorimotor processes, and at the later stage, the explicit sense of agency emerges and can be modified by reflective processes (e.g., retrospection). Thus, as implicit and explicit levels of sense of agency might be different but spring from the same origin, both levels are apparently connected and are presumably correlated. Intuitively, when individuals experience either explicit or implicit aspects of agency, they may be likely to also experience the other aspect of agency. However, to our knowledge, studies have reported only inter-individual correlations between trends in agency judgment and intentional binding, postulating that people who experience a stronger or weaker explicit sense of agency than others also tend to experience stronger or weaker intentional binding. Statistically speaking, inter-individual variation is rarely equal to intra-individual variation (Molenaar \& Campbell, 2009). Thus, findings on inter-individual correlations between two aspects of agency cannot be applied to the discussion of intra-individual correlation. A fundamental and unanswered 
question related to intra-individual correlation is whether strong or weak explicit sense of agency coincides with strong or weak intentional binding within individuals (e.g., on a trial-by-trial basis). It has been argued that discussion of the relationship between explicit and implicit measures is itself unproductive (Fazio \& Olson, 2003) because of strong dependence on operational definitions of the construct and methodological constraints. Nevertheless, investigation of intra- and inter-individual variations between explicit and implicit measures of sense of agency may be beneficial, because to date, the mechanism of intentional binding and the relationships between sense of self, voluntary action, and time perception are yet to be fully understood (Merchant \& Yarrow, 2016).

\subsection{The present study}

To our knowledge, this is the first study to examine whether explicit sense of agency and intentional binding (i.e., implicit measure of sense of agency) intra-individually correlate on a trial-by-trial basis. Inter-individual correlations were also examined for comparison with previous findings (e.g., Ebert \& Wegner, 2010). Another novel aspect of the present study involved testing the intra- and inter-individual correlations in two experiments employing auditory (Experiment 1) and visual action-outcomes (Experiment 2) to examine reproducibility and any modality-specificity of the expected correlations. The present experiments employed an established paradigm (e.g., Dewey \& Knoblich, 2014; Humphreys \& Buehner, 2009) where, in the "action" task, participants voluntarily pressed a key that was followed by auditory outcome (i.e., pip tone) with five levels of delay ranging from $100 \mathrm{~ms}$ to $900 \mathrm{~ms}$. The range of outcome delays were intended to induce varying degrees of sense of agency within individuals, in order to examine intra- and inter-individual correlations between measures of sense of agency. We expected that larger delays would cause weaker sense of agency, and vice versa, in line with previous findings (e.g., Sato \& Yasuda, 2005). In each trial, participants were required to rate the perceived sense of agency and estimate the subjective outcome delay. Participants performed another "baseline" task, where they passively listened to one tone followed by another tone with the same range of delays as the action task. The estimated delays in the baseline task were used as reference values to calculate the degree of intentional binding under each outcome-delay condition. We expected that larger delays would cause less intentional binding, consistent with previous findings (Haggard et al., 2002), and that variability due to the outcome delay (i.e., the regression slope) would "inter-individually" correlate between agency rating and the degree of intentional binding. Finally, of primary interest to this study, we also expected that agency ratings and intentional binding would "intra-individually" correlate on a trail-by-trial basis regardless of the delay conditions if intentional binding and explicit sense of agency coincide. 


\section{Experiment 1}

\subsection{Material and methods}

\subsubsection{Participants}

Thirty-four Japanese undergraduates (18 females; mean age 19.29 years, standard deviation $(S D)=$ 0.76) participated in return for monetary compensation. Sample size was determined based on a previous study on auditory and visual temporal binding (Fereday \& Buehner, 2017), and a priori power analysis with G*Power 3.1.9.3 (Faul, Erdfelder, Lang, \& Buchner, 2007) for a correlation analysis. The power analysis indicated that 34 participants were required for a statistical power of 0.90 , assuming a large effect size of 0.50 (Cohen, 1988) and Type I error probability of 0.05 .

All participants reported that they were right-handed without a history of neurological or psychiatric disorders and had normal or corrected-to-normal visual acuity. To confirm that our participants had normal experiences regarding the sense of self on an everyday basis, participants completed the Embodied Sense of Self Scale (ESSS) (Asai, Kanayama, Imaizumi, Koyama, \& Kaganoi, 2016), which consists of three subscales: Agency (Mean $=23.65, S D=4.95)$, Ownership (Mean $=19.47, S D$ $=4.94$ ), and Narrative (Mean $=27.29, S D=5.08$ ). We calculated the effect size (Cohen's $d$ ) of the difference between mean scores in the present sample and a healthy sample from a previous study $(N$ $=96,36$ females; mean age 22.55 years) (Kanayama et al., 2017). The effect sizes $(d \mathrm{~s})$ were small: 0.223 for Agency, 0.481 for Ownership, and 0.479 for Narrative, suggesting that our sample were normal in terms of daily experiences of the sense of self. Each participant provided written informed consent prior to the experiment. The present study was conducted in accordance with the Declaration of Helsinki and was approved by the local ethical committee of the Graduate School of Arts and Sciences, The University of Tokyo (approval number: 521).

\subsubsection{Apparatus and stimuli}

Auditory stimuli were two pure tones with pitches of 600 and $1000 \mathrm{~Hz}$ (low and high tones, respectively), at a constant comfortable volume, and $100 \mathrm{~ms}$ in duration. Tones were presented via headphones (QuietComfort 25, Bose, Framingham, Massachusetts). A fixation cross “+” subtending a visual angle of approximately $0.8^{\circ}$, as well as all instructions, questions, and response scales were white-colored and presented against a black background on a 13.3-inch liquid crystal display monitor built in to a computer (R63/PS, Toshiba, Tokyo, Japan) with a resolution of $1920 \times 1080$ pixels and refresh rate of $60 \mathrm{~Hz}$. Participants viewed the monitor at a distance of approximately $60 \mathrm{~cm}$ without a chin rest. Participants' responses were collected via an external keypad (BSTK07, Buffalo, Nagoya, Japan). Stimulus presentation and response collection were controlled by the computer with a custom program written in Hot Soup Processor 3.4 (ONION Software, Japan) running on Windows 7 
Professional 64-bit (Microsoft, Redmond, Washington).

\subsubsection{Tasks and procedures}

In a trial of the "action" task (Fig. 1), following the presentation of the fixation cross on the monitor, participants voluntarily pressed the "enter" key on the keypad using their right index finger. The high tone was presented through the headphones $100,300,500,700$, or $900 \mathrm{~ms}$ after the keypress. The fixation cross remained for approximately $400 \mathrm{~ms}$ (randomly jittered between 300 and $500 \mathrm{~ms}$ ) after the high tone, to ensure that the action outcome was the high tone and not the disappearance of the fixation cross. Finally, the monitor presented a question asking participants to rate their perceived sense of agency over the tone ("How much did you feel that you caused the tone?") using a 9-point Likert scale ranging from 1 ("Not at all") to 9 ("Extremely strongly"). This question was adapted from previous studies on the relationship between explicit and implicit measures of agency (Dewey \& Knoblich, 2014; Ebert \& Wegner, 2010; Pyasik et al., 2018; Wen, Yamashita, \& Asama, 2015). Participants responded to the question using the keypad without any time pressure. Their response was presented on the monitor as they typed and could be amended before finalization. Next, a statement appeared on the monitor asking participants to estimate the action-outcome delay ("Estimate the delay between the keypress and the tone using 1 to 1000 (ms)"). Participants were informed that the delay varied randomly between 1 and $1000 \mathrm{~ms}$ on a trial by trial basis and were allowed to use all integers between 1 and 1000 . No feedback on the accuracy of their delay estimation was provided. Verbal estimation of temporal interval has been validated in studies on intentional binding (Caspar, Cleeremans, \& Haggard, 2015; Engbert et al., 2008; Engbert et al., 2007; Fereday \& Buehner, 2017; Humphreys \& Buehner, 2009). The order of the two questions was fixed within participants and counterbalanced across participants. Inter-trial interval was approximately $800 \mathrm{~ms}$ (jittered between 700 and $900 \mathrm{~ms}$ ).

The "baseline" task (Fig. 1) was identical to the action task except that participants passively listened to a low-pitched tone instead of performing a voluntary action (i.e., a keypress). A pilot experiment with an additional 12 healthy undergraduates who performed 60 trials of the action task showed that they pressed the enter key on average $703.6 \mathrm{~ms}$ (standard error of the mean 69.7) after the onset of the initial fixation cross. To balance the timings of the baseline and action tasks, the initial presentation of the fixation cross in the baseline task lasted for approximately $700 \mathrm{~ms}$ (jittered between 600 and 800 ms). Next, the low tone was presented followed by the high tone with five levels of delay $(100,300$, 500,700 , or $900 \mathrm{~ms}$ ). Participants responded only to a question asking them to estimate the outcome delay ("Estimate the delay between two tones using 1 to 1000 (ms)"), using the same verbal estimation method as in the action task. 


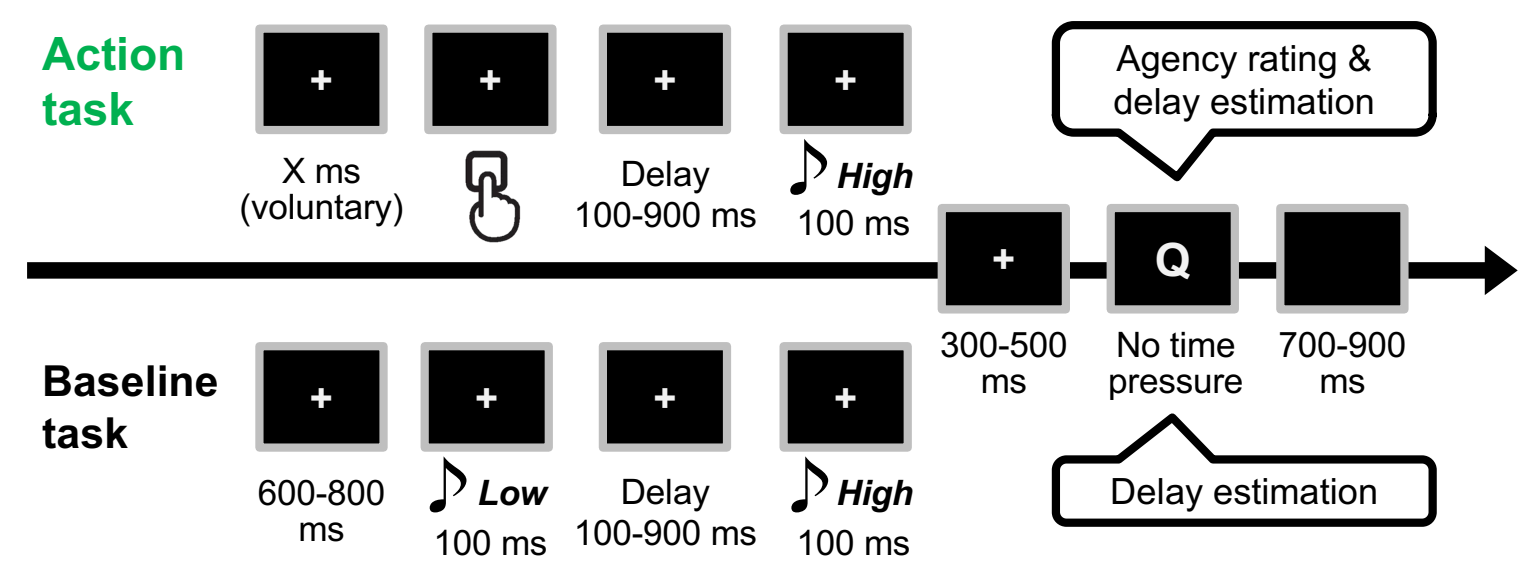

Fig. 1. Schematic illustration of a trial of the action or baseline task in Experiment 1.

The experiment was conducted in a quiet and well-lit room. Participants removed any wrist and hand jewelry including watches, wore the headphones, and performed a practice session which consisted of two blocks including six trials each of action and baseline tasks. In the practice, only three delays of 100, 500, and $900 \mathrm{~ms}$ were used twice for each block. In the main session following the practice, the action and baseline tasks were blocked in an $\mathrm{ABAB}$ order. Participants performed either action or baseline tasks over seven trials each with five outcome delays per block, resulting in 140 trials in total. The order of blocks was counterbalanced across participants. The order of outcome delays was randomized within a block.

\subsubsection{Data analysis}

Agency ratings in action task and estimated delays in action and baseline tasks under each outcome-delay condition were averaged for each participant. To check the effect of outcome delays on the perceived sense of agency, analysis of variance (ANOVA) with a within-participant factor of outcome delay (five levels) was performed on the agency ratings. To check whether intentional binding occurred (i.e., shorter delay estimates in action tasks), ANOVA with two within-participant factors of task (action versus baseline) and outcome delay (five levels) was performed on the estimated delays. Finally, to examine the effect of outcome delays on the degree of intentional binding, we calculated estimated-delay ratios by dividing the estimated delays in the baseline task by those in the action task. A ratio larger than 1.0 corresponds to shorter delay estimation in the action task and vice versa. Therefore, larger ratios suggest stronger intentional binding. The ratios were first compared with 1.0 using two-tailed one-sample tests for each delay condition with the false discovery rate correction for multiple tests (Benjamini \& Hochberg, 1995). Given that the ratios, but not the 
absolute differences, enable comparison of estimated durations between conditions using different standard durations (Block \& Zakay, 1997; Hornstein \& Rotter, 1969), we employed the estimated-delay ratio as an index of the degree of intentional binding to be compared across the five delay conditions using ANOVA. When the sphericity assumption for ANOVA was violated, Greenhouse-Geisser correction was applied to the degrees of freedom. Bonferroni correction was used for multiple comparisons in post-hoc tests for ANOVA.

To examine whether explicit sense of agency coincides with intentional binding while being modulated by action-outcome temporal (in)congruence, we analyzed inter-participant correlation between linear-regression slopes over agency ratings and those over estimated-delay ratios (i.e., degree of intentional binding). Positive correlation would indicate that agency and intentional binding covary with the outcome delays. Linear regressions with five levels of outcome delay as a regressor were fitted separately to agency rating and estimated-delay ratio in 70 trials of the action task for each participant using the least-squares method. The estimated-delay ratio for each trial in the action task was calculated by referring to estimated delays averaged for each outcome-delay condition in the baseline task. Note that outcome delays as a regressor were handled in units of seconds for simplicity of output values.

To further check the coincidence of explicit sense of agency and intentional binding, we analyzed the effect size of intra-participant correlations between agency ratings and estimated-delay ratios in 70 action-task trials for each participant. Positive correlation indicates that stronger (weaker) sense of agency coincides with a larger (smaller) degree of intentional binding on a trial by trial basis. We employed Spearman's rank correlation coefficient rho $(\rho)$ because of its robustness to outlying data and the nature of agency ratings as an ordinal scale. We then estimated $95 \%$ confidence intervals (CI) of the averaged correlation coefficient using the bootstrapped resampling method with 10,000 iterations.

Finally, we checked if there were any (interactive) effects of the question order of agency rating and interval estimation as a between-participants factor on the above dependent and independent variables in ANOVA. We also tested the group differences in the regression slopes over agency rating and estimated-delay ratio and the intra-individual correlation coefficients using two-tailed $t$-test.

ANOVA was conducted using SPSS 24.0 (IBM Corp., Armonk, New York). Linear regression, correlations analysis, and bootstrapping estimations were conducted using R 3.4.4 (R Core Team, 2018). In addition, we used the R packages simpleboot (Peng, 2008) for bootstrapping and ggplot2 
(Wickham, 2009) for visualization.

\subsection{Results and discussion}

Fig. 2A shows the results of subjective rating of perceived sense of agency over auditory outcomes (for detailed values, see Table A.1). We found significant effect of outcome delay on the agency rating $\left(F(1.87,61.66)=170.02, p<0.001, \eta_{\mathrm{p}}^{2}=0.837\right)$. Post-hoc comparisons indicate significant differences between each adjacent delay condition $(p s<0.001)$. As expected, the reported sense of agency over the auditory outcome decreased with increasing outcome delay, consistent with previous findings (Asai \& Tanno, 2008; Sato \& Yasuda, 2005). Outcome delays can violate the prediction of spatio-temporally matched sensory consequences by internal forward models, resulting in the failure to generate sense of agency.

Fig. 2B displays the results of verbal estimation of the outcome delays. ANOVA revealed that main effects of outcome delay $\left(F(1.22,40.11)=262.36, p<0.001, \eta_{\mathrm{p}}^{2}=0.888\right)$ and task $(F(1,33)=31.81$, $\left.p<0.001, \eta_{\mathrm{p}}^{2}=0.491\right)$ on the estimated delays. There was no interaction between two factors $(F(2.74$, $\left.90.39)=0.45, p=0.701, \eta_{\mathrm{p}}^{2}=0.013\right)$. Post-hoc tests revealed significant differences between each adjacent delay condition ( $p$ s $<0.001$ ), suggesting that participants were able to discriminate all levels of outcome delay. Importantly, participants reported shorter estimated-delay in the action task than in the baseline task under all delay conditions $(p s<0.001)$, suggesting that intentional binding was replicated in the current sample and experimental design, and emerged regardless of action-outcome temporal contiguity.

Nevertheless, a remaining question is whether the "degree" of intentional binding was affected by the outcome delay. Fig. $2 \mathrm{C}$ displays the estimated-delay ratio as an index of the degree of intentional binding. We began by confirming that the ratios for all delay conditions significantly differed from 1.0 $(t \mathrm{~s}(33)>4.67, p \mathrm{~s}<0.001, d \mathrm{~s}>0.800)$, again indicating the emergence of intentional binding. It seems that the ratio decreased with increasing outcome delays. Indeed, there was a significant effect of outcome delay upon estimated-delay ratio $\left(F(1.16,38.37)=20.06, p<0.001, \eta_{\mathrm{p}}{ }_{\mathrm{p}}=0.378\right)$. Post-hoc tests showed differences between all adjacent delay conditions $(p s<0.019)$ except for between the 500 and $700 \mathrm{~ms}$ conditions $(p=0.999)$. These results indicate that intentional binding requires temporal contiguity between an action and its outcome, and thus can be attenuated by temporal discrepancy between them, in line with a previous finding (Haggard et al., 2002). Furthermore, variation of intentional binding by outcome delay may reflect the nature of explicit aspect of sense of agency (e.g., rating), as discussed above. 
A

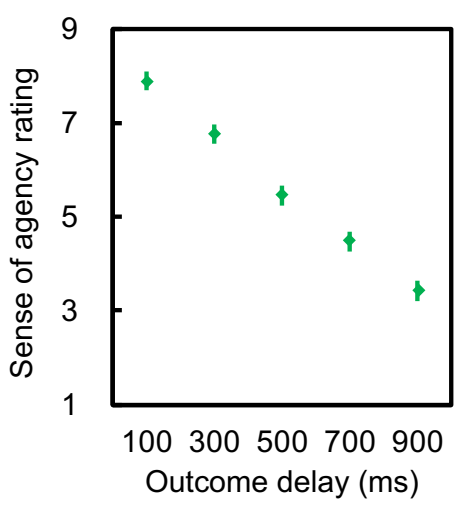

B

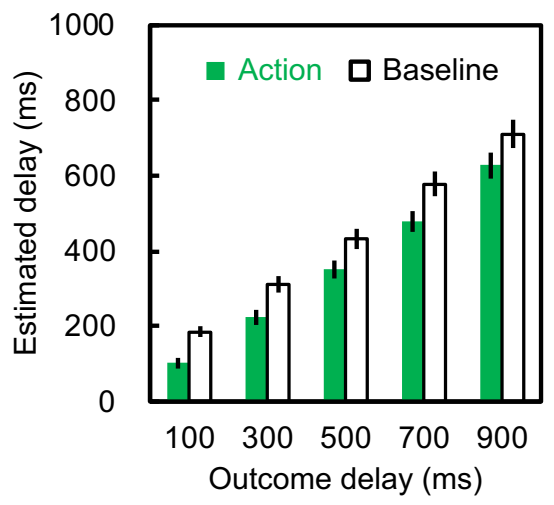

C

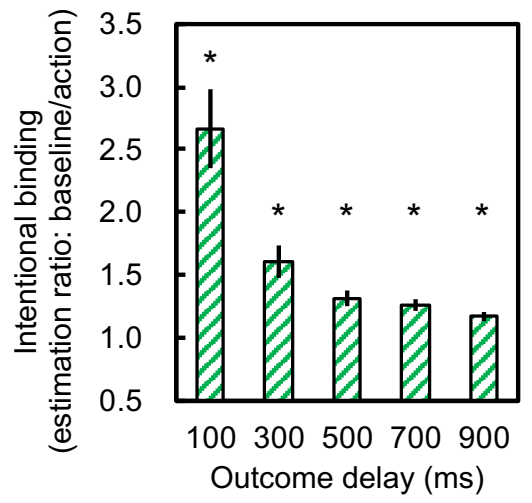

Fig. 2. Results from Experiment 1: (A) Mean rating score of sense of agency over a tone as a function of actual outcome delay. Larger rating scores indicate stronger sense of agency. (B) Mean estimated outcome delay in action and baseline tasks as a function of their actual delay. (C) Mean ratio of the estimated delay in the baseline task to that in the action task (i.e., intensity of intentional binding) as a function of the actual outcome delay. Larger ratios indicate greater intentional binding. Error bars denote one standard error of the mean. Asterisks indicate significant differences between the mean score and $1.0(p<0.01)$.

Fig. 3A displays a scatterplot of the linear-regression slopes for agency rating and intentional binding. Since slopes for intentional binding were not normally distributed (Shapiro-Wilk's $W=0.964, p=$ 0.001), we calculated Spearman's rank correlation coefficient, resulting in a significant positive correlation between the two slopes $(\rho=0.372,95 \%$ CI $[0.039,0.631], p=0.030)$. Therefore, intentional binding and agency rating might be similarly modulated by action-outcome temporal contiguity.

A

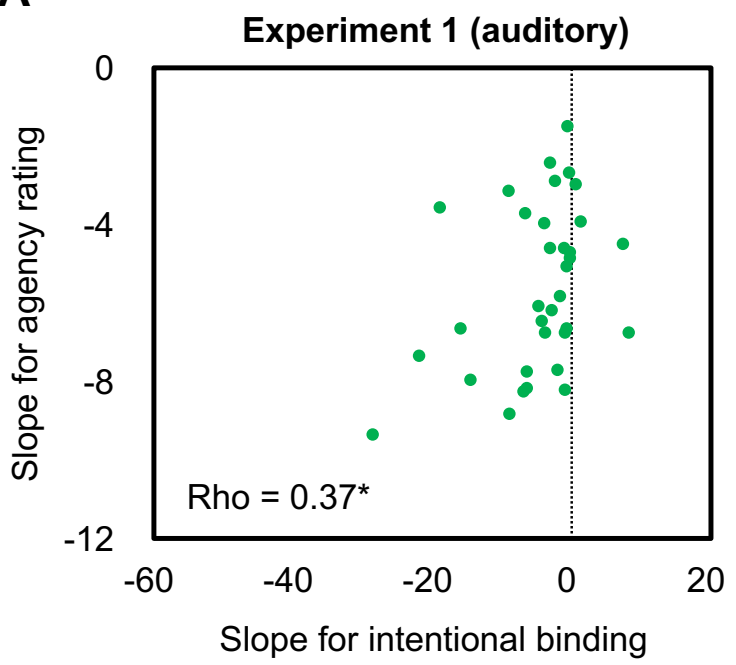

B

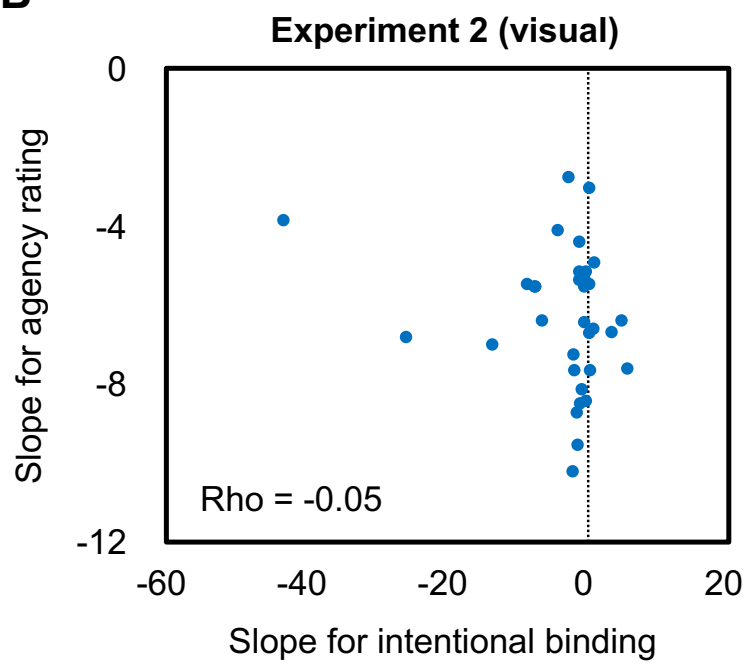


Fig. 3. Relationship between slopes of linear regression of sense of agency rating and intentional binding intensity in (A) Experiment 1 and (B) Experiment 2. Rank correlation coefficients (Spearman's rho) were reported because of the non-normality in the slopes for intentional binding. Asterisk indicates statistical significance $(p<0.05)$.

We calculated intra-individual correlations between intentional binding intensity and agency rating on a trial by trial basis regardless of the outcome delays. As shown in Fig. 4, rank correlation coefficients from 34 participants ranged from 0.016 to 0.912 and were normally distributed ( $W=0.964, p=0.312$ ). We estimated the average correlation coefficients as 0.577 with bootstrapped $95 \%$ CI from 0.505 to 0.648. This result suggests that intentional binding (i.e., estimated-delay ratio) and agency rating are positively correlated even within individuals. Therefore, to tentatively conclude, intentional binding coincides with explicit sense of agency while being modulated by action-outcome (in)congruence.

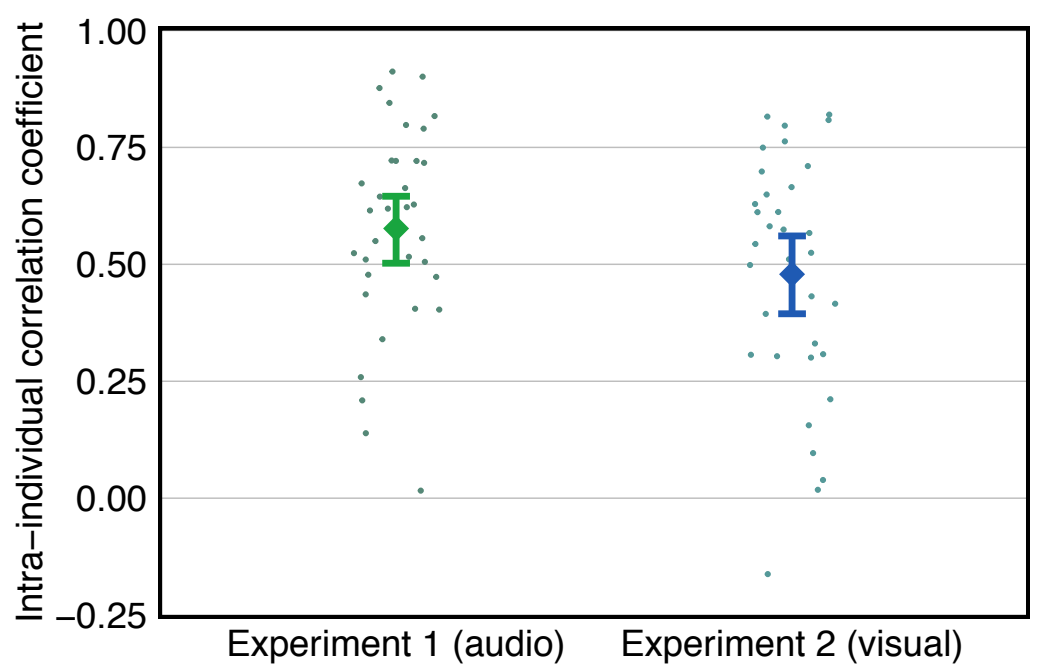

Fig. 4. Intra-individual rank correlations (Spearman's rho) between agency rating and intentional binding in 70 trials of the action task. Each dot indicates each participant in Experiments 1 and 2. Square markers and error bars denote averaged coefficients and $95 \%$ bootstrapped confidence intervals, respectively.

Potential effects of the order of two questions were examined. We found a significant main effect of question order on the agency rating $\left(F(1,32)=6.30, p=0.017, \eta_{\mathrm{p}}^{2}=0.164\right)$. Participants who rated the agency at first (mean across the delay conditions $=5.21, \mathrm{SD}=0.99$ ) scored lower than those who estimated the outcome delay at first (mean $=6.00, \mathrm{SD}=0.84$ ). Importantly, there was no interaction between question order and outcome delay $\left(F(1.83,58.73)=0.38, p=0.671, \eta_{\mathrm{p}}^{2}=0.012\right)$. We found neither main effect of question order on interval estimation $\left(F(1,32)=1.15, p=0.292, \eta_{\mathrm{p}}{ }_{\mathrm{p}}=0.035\right)$, nor interaction with outcome delay $\left(F(1.21,38.77)=0.27, p=0.649, \eta_{\mathrm{p}}^{2}=0.008\right)$, nor interaction 
with task $\left(F(1,32)=1.06, p=0.311, \eta_{\mathrm{p}}^{2}=0.032\right)$, nor interaction between question order, outcome delay, and task $\left(F(2.71,86.73)=1.19, p=0.317, \eta^{2}=0.036\right)$. As for the estimated-delay ratios, there were neither main effect of question order $\left(F(1,32)=2.66, p=0.113, \eta_{\mathrm{p}}^{2}=0.077\right)$ nor interaction with outcome delay $\left(F(1.17,37.42)=1.46, p=0.239, \eta_{\mathrm{p}}^{2}=0.044\right)$. Most importantly, we found no differences between question orders in the regression slopes for agency rating $(t(32)<0.01, p=0.998$, $d<0.001)$ and estimated-delay ratios $(t(32)=0.53, p=0.601, d=0.181)$, and the intra-individual correlation coefficients $(t(32)=-1.27, p=0.213, d=-0.436)$. In sum, results showed that, although the question order had an effect on the agency ratings, it did not interact with the other factors and did not affect the relationships between agency rating and estimated-delay ratio (i.e., intentional binding).

\section{Experiment 2}

Experiment 1 suggested an intra-individual correlation between intentional binding and subjective rating of sense of agency that varied with action-outcome temporal congruence. To investigate any modality-specificity in our findings, Experiment 2, using newly recruited healthy adults, was conducted employing the same experimental design as Experiment 1 except that "visual" action outcomes (i.e., presentation of static circle) were used instead of tones. It has been shown that visual feedback on action is able to induce sense of agency which can be modulated by temporal congruence between action and the feedback based on the comparator mechanism (Farrer, Bouchereau, Jeannerod, \& Franck, 2008; Farrer et al., 2013; Franck et al., 2001; Imaizumi \& Asai, 2017). Moreover, intentional binding has also been observed in the visual domain (Ebert \& Wegner, 2010; Engbert et al., 2008), but can be weaker than in the auditory domain (Ruess et al., 2018). We expected that in this experiment, subjective ratings of agency over visual outcomes, similar to auditory outcomes, would be modulated by action-outcome temporal congruence (i.e., outcome delays). On the other hand, modality-specificity might be observed, in that visual intentional binding would be weakened so as to be less sensitive to outcome delays. If this is the case, correlations between regression slopes for ratings and intentional binding would also be weakened. Nevertheless, regardless of sensitivity to outcome delay, agency rating and intentional binding in the visual domain may be expected to intra-individually correlate on a trial by trial basis.

\subsection{Material and methods}

\subsubsection{Participants}

Thirty-five newly recruited Japanese undergraduates participated. One participant was excluded from the analysis because of a technical problem during the experiment. We thus analyzed the remaining 34 participants (17 females; mean age 19.50 years, $S D=0.99$ ). All reported that they were right-handed without a history of neurological or psychiatric disorders and had normal or corrected-to-normal 
visual acuity. This sample was comparable with Experiment 1 in terms of age (non-normal distribution; Mann-Whitney's $\mathrm{U}=521.5, p=0.465$, rank biserial correlation $\left.\left(\rho_{\mathrm{rb}}\right)=0.098\right)$ and sex $\left(\chi^{2}(1)=0.06, p=0.808, \varphi=0.029\right)$, as well as everyday experiences of the sense of self measured by the ESSS: Agency $($ Mean $=25.44, S D=4.72 ; t(66)=1.53, p=0.131, d=0.371)$, Ownership (Mean = $21.50, S D=6.17 ; t(66)=1.50, p=0.139, d=0.363)$, and Narrative $($ Mean $=28.24, S D=4.98 ; t(66)=$ $0.77, p=0.443, d=0.187)$.

\subsubsection{Apparatus and stimuli}

Instead of the auditory stimuli used in Experiment 1, gray- and blue-colored circles with the same luminance lasting for $100 \mathrm{~ms}$ were used as visual cue and visual feedback on keypress (Fig. 5). The circles, which were drawn with a thickness of $0.2^{\circ}$ of visual angle, subtended $2.4^{\circ}$ of diameter at the center of the monitor so as to surround the fixation cross which subtended $0.8^{\circ}$. Visual stimuli and white-colored text (i.e., instructions, questions, and response scales) were presented against a black background on a 24-inch liquid crystal display monitor (V242, Hewlett Packard, Palo Alto, California) with a resolution of $1920 \times 1080$ pixels and refresh rate of $60 \mathrm{~Hz}$. Participants viewed the monitor at a distance of $60 \mathrm{~cm}$ with a chin rest. Participants' responses were collected via the same keypad as used in Experiment 1. Stimulus presentation and response collection were controlled by a computer with a custom program written in Hot Soup Processor 3.4 (ONION Software, Japan) running on Windows 10 Professional 64-bit (Microsoft, Redmond, Washington).

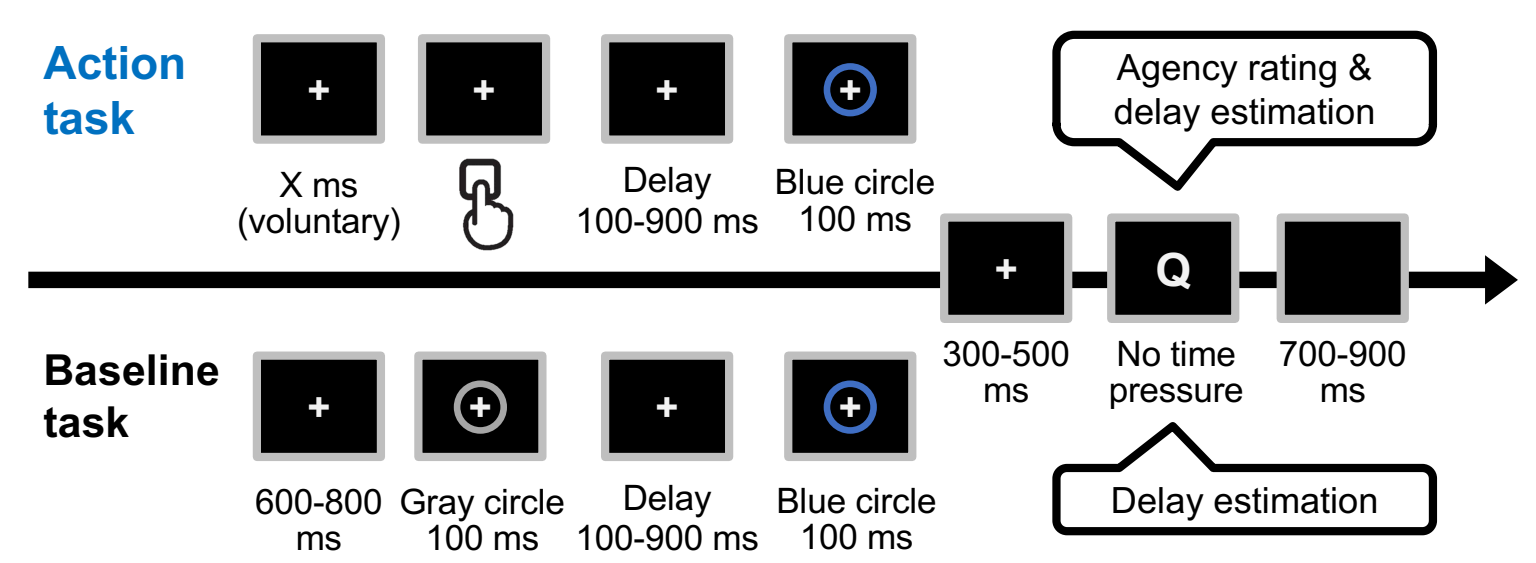

Fig. 5. Schematic illustration of a trial of the action or baseline task in Experiment 2.

\subsubsection{Tasks and procedures}

The tasks were identical to those in Experiment 1 except that the auditory stimuli were replaced with visual stimuli (i.e., two equiluminant circles) and accordingly questions were also modified (Fig. 5). In the action task, the blue circle was presented on the monitor 100, 300, 500, 700, or $900 \mathrm{~ms}$ after a 
voluntary keypress. Then, the monitor presented a question asking participants to rate the perceived sense of agency ("How much did you feel that you caused the blue circle?") with the same 9-point Likert scale as Experiment 1. Next, another statement asking participants to estimate the outcome delay was presented ("Estimate the delay between the keypress and the circle using 1 to 1000 (ms)"). Verbal estimation of temporal interval has also been validated for measurement of visual intentional binding (Engbert et al., 2008; Fereday \& Buehner, 2017). In the baseline task, following the initial presentation of the fixation cross, the gray circle was presented followed by the blue circle with the five levels of delay. Participants responded to a question asking them to estimate the circle-circle delay ("Estimate the delay between two circles using 1 to 1000 (ms)") by using the same verbal estimation method. Block design and trial repetitions for the practice and main sessions were identical to those in Experiment 1.

\subsubsection{Data analysis}

Analysis procedures and software were identical to those in Experiment 1. In addition, cross-experiment analysis performed by adding a between-factor (i.e., Experiment 1 versus 2 ) to the above ANOVAs for the measures of agency rating, delay estimation, and the estimated-delay ratio. Furthermore, intra-individual correlations between agency rating and intentional binding were compared between experiments by using a two-tailed unpaired $t$-test.

\subsection{Results and discussion}

\subsubsection{Sense of agency in the visual domain}

Subjective ratings of the sense of agency over visual outcomes decreased with increasing outcome-delays (Fig. 6A). There was a significant effect of outcome delay $(F(2.03,66.87)=269.15, p$ $\left.<0.001, \eta_{\mathrm{p}}^{2}=0.891\right)$ and significant differences between all adjacent delay conditions $(p \mathrm{~s}<0.001)$. These results were expected given that outcome delay can violate the prediction of spatio-temporally matched sensory feedback on one's voluntary action, resulting in a decreased sense of agency (Franck et al., 2001; Sato \& Yasuda, 2005).

As for delay estimation (Fig. 6B), ANOVA revealed main effects of outcome delay $(F(1.38,45.61)=$ $\left.380.76, p<0.001, \eta_{\mathrm{p}}^{2}=0.920\right)$ and significant differences between all adjacent delay conditions $(p \mathrm{~s}<$ 0.001 ), suggesting that participants could discriminate the five levels of outcome delay. However, we did not find a main effect of task $\left(F(1,33)=0.92, p=0.346, \eta_{\mathrm{p}}^{2}=0.027\right)$, nor an interaction between the two factors $\left(F(2.52,82.99)=1.10, p=0.349, \eta_{\mathrm{p}}^{2}=0.032\right)$. No significant differences between action and baseline tasks might suggest that there was no intentional binding for visual outcomes regardless of the actual outcome delays. Nevertheless, we performed one-sample tests comparing 
estimated-delay ratios with 1.0 (i.e., equal estimation in action and baseline tasks) to determine whether intentional binding occurred under each delay conditions. As shown in Fig. 6C, intentional binding became evident in the small delay conditions. Since estimated-delay ratios under 100, 300, and $700 \mathrm{~ms}$ delay conditions were not normally distributed $(W \mathrm{~s}<0.908, p \mathrm{~s}<0.008)$, we performed two-tailed one-sample Wilcoxon signed-rank tests with the false discovery rate correction for multiple tests. Ratios significantly higher than 1.0 were found in the 100 and $300 \mathrm{~ms}$ delay conditions $(100 \mathrm{~ms}$ : $\left.V=439, p=0.038, \rho_{\mathrm{rb}}=0.476 ; 300 \mathrm{~ms}: V=477, p=0.010, \rho_{\mathrm{rb}}=0.603\right)$ but not in the other conditions with larger actual delays (500 ms: $V=414, p=0.077, \rho_{\mathrm{rb}}=0.392 ; 700 \mathrm{~ms}: V=332, p=$ $\left.0.566, \rho_{\mathrm{rb}}=0.116 ; 900 \mathrm{~ms}: V=339, p=0.566, \rho_{\mathrm{rb}}=0.139\right)$. The ANOVA indicated a significant effect of outcome delay on estimated-delay ratio $\left(F(1.44,47.61)=5.69, p=0.012, \eta_{\mathrm{p}}^{2}=0.147\right)$, although post-hoc tests showed no differences between all adjacent delay conditions $(p s>0.149)$. These results suggest that visual intentional binding can occur only when the action-outcome temporal discrepancy is smaller than $500 \mathrm{~ms}$. Interestingly, this temporal limit of visual intentional binding was consistent with previous findings (Ebert \& Wegner, 2010; Engbert et al., 2008; Ruess et al., 2018).
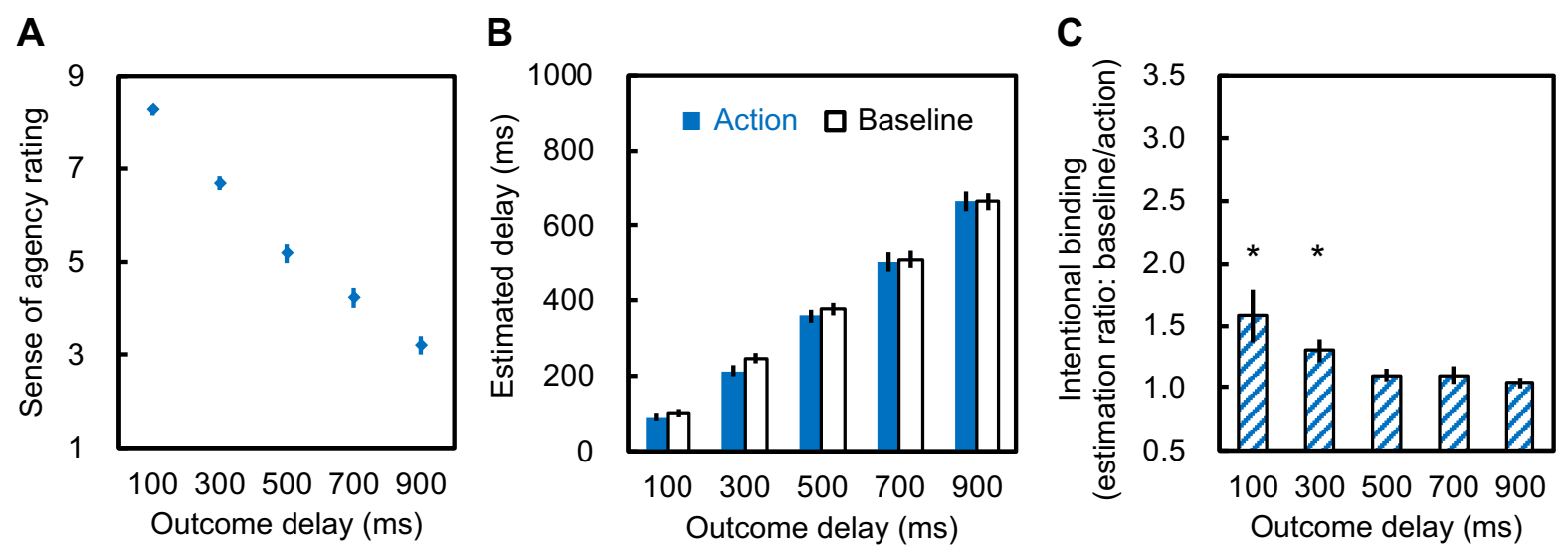

Fig. 6. Results from Experiment 2: (A) Mean rating score of sense of agency over visual feedback (i.e., blue circle) as a function of actual outcome delay. Larger rating scores indicates stronger sense of agency. (B) Mean estimated outcome delay in action and baseline tasks as a function of their actual delay. (C) Mean ratio of the estimated delay in the baseline task to that in the action task (i.e., intentional binding) as a function of the actual delay. Larger ratios indicate greater intentional binding. Error bars denote standard error of the mean. Asterisks indicate significant differences between the mean score and $1.0(p<0.05)$.

As shown in Fig. 3B, there was no correlation between regression slopes for agency rating and intentional binding ( $\rho=-0.049,95 \%$ CI $[-0.381,0.294], p=0.783$ : note that the slope for binding was non-normally distributed, $W=0.611, p<0.001)$. It is possible that this was because slopes for visual intentional binding crowded around zero (i.e., almost insensitive to actual outcome delays). We can 
indeed see this tendency in the figure and accordingly find that the median slope for Experiment 2 was -1.17 with an interquartile range of -3.18 to 0.15 , compared to -2.89 (interquartile range -6.64 to -0.60) for Experiment 1. Less inter-individual variation in regression slopes and insensitivity to modulation by the outcome delays for visual intentional binding might have resulted in the null inter-individual correlation between intentional binding and agency rating. Therefore, we analyzed "intra-individual" correlation between intentional binding and agency rating in 70 trials regardless of the actual outcome delays. As shown in the right column in Fig. 4, rank correlation coefficients from each participant ranged from -0.162 to 0.820 (normally distributed: $W=0.946, p=0.092$ ). We estimated the average correlation coefficient as 0.479 with bootstrapped $95 \%$ CI from 0.398 to 0.562 , suggesting that the degree of intentional binding and agency rating are positively correlated within individuals. Even though we did not observe inter-individual correlation in terms of regression slopes, intentional binding and explicit sense of agency over visual outcomes may in fact coincide within individuals on a trial by trial basis.

The question order did not affect the above results. We found neither main effect of question order on the agency rating $\left(F(1,32)=0.06, p=0.804, \eta_{\mathrm{p}}^{2}=0.002\right)$ nor interaction with outcome delay $(F(2.00$, $\left.64.08)=0.26, p=0.776, \eta_{\mathrm{p}}^{2}=0.008\right)$. There was neither main effect of question order on interval estimation $\left(F(1,32)=0.20, p=0.659, \eta_{\mathrm{p}}^{2}=0.006\right)$, nor interaction with outcome delay $(F(1.37$, $\left.43.89)=0.22, p=0.716, \eta_{\mathrm{p}}^{2}=0.007\right)$, nor interaction with task $\left(F(1,32)=1.70, p=0.202, \eta_{\mathrm{p}}^{2}=\right.$ $0.050)$, nor interaction between question order, outcome delay, and task $(F(2.51,80.19)=0.17, p=$ $\left.0.884, \eta_{\mathrm{p}}^{2}=0.005\right)$. As for the estimated-delay ratios, there were neither main effect of question order $\left(F(1,32)=2.26, p=0.143, \eta^{2} \mathrm{p}=0.066\right)$ nor interaction with outcome delay $(F(1.47,47.00)=1.56, p$ $\left.=0.223, \eta_{\mathrm{p}}^{2}=0.046\right)$. We found no differences between question orders in the regression slopes for agency rating $(t(32)=-0.04, p=0.970, d=-0.013)$ and estimated-delay ratios $(t(32)=-0.38, p=$ $0.706, d=-0.131)$, and the intra-individual correlation coefficients $(t(32)=-1.70, p=0.098, d=$ $-0.585)$.

\subsubsection{Cross-experiment analysis: auditory versus visual outcomes}

Ratings of sense of agency over sensory outcomes decreased with the increasing outcome delays consistently in the two experiments. As expected, cross-experiment ANOVA revealed neither a main effect of experiment $\left(F(1,66)=0.19, p=0.664, \eta_{\mathrm{p}}^{2}=0.003\right)$ nor an interaction with outcome delay $\left(F(1.98,130.91)=2.06, p=0.086, \eta_{\mathrm{p}}^{2}=0.030\right)$, suggesting that explicit sense of agency is modulated by action-outcome temporal congruence without audio-visual specificity.

Importantly, delay estimation and the ratio between action and baseline tasks might be 
modality-specific, as we find evidence for weaker intentional binding in the visual domain. Regarding the delay estimation, ANOVA revealed neither a main effect of experiment $(F(1,66)=1.08, p=0.302$, $\left.\eta_{\mathrm{p}}^{2}=0.016\right)$, nor an interaction between experiment and outcome delay $(F(1.32,86.83)=0.89, p=$ $\left.0.377, \eta_{\mathrm{p}}^{2}=0.013\right)$, nor an interaction between experiment, outcome delay, and task $(F(2.66,175.40)$ $\left.=0.90, p=0.431, \eta_{\mathrm{p}}^{2}=0.014\right)$. However, we did find a significant interaction between experiment and task on delay estimation $\left(F(1,66)=12.26, p=0.001, \eta_{\mathrm{p}}^{2}=0.157\right)$. A simple main effect of task was found in Experiment $1\left(F(1,66)=34.44, p<0.001, \eta_{\mathrm{p}}^{2}=0.343\right)$, but not in Experiment $2(F(1,66)=$ $\left.0.84, p=0.363, \eta_{\mathrm{p}}^{2}=0.013\right)$. These results suggest that intentional binding in terms of a "difference" between action and baseline tasks was found only in auditory domain (i.e., Experiment 1).

As for the estimated-delay ratio, there were significant main effects of outcome delay $(F(1.26,82.84)$ $\left.=25.31, p<0.001, \eta_{\mathrm{p}}^{2}=0.277\right)$, experiment $\left(F(1,66)=9.81, p=0.003, \eta_{\mathrm{p}}^{2}=0.129\right)$, and their interaction $\left(F(4,264)=5.94, p<0.001, \eta^{2}=0.083\right)$. A simple main effect of outcome delay was found in Experiment $1\left(F(4,63)=9.02, p<0.001, \eta_{\mathrm{p}}^{2}=0.364\right)$ but not in Experiment $2(F(4,63)=$ $\left.2.01, p=0.103, \eta_{\mathrm{p}}^{2}=0.113\right)$. This result supports the view that visual intentional binding is insensitive to outcome delays as shown by regression slopes around zero in Experiment 2 (Fig. 3B). Furthermore, there were (marginally) significant simple main effects of experiment in all delay conditions (100 ms: $F(1,66)=8.39, p=0.005, \eta_{\mathrm{p}}^{2}=0.113 ; 300 \mathrm{~ms}: F(1,66)=3.97, p=0.051, \eta_{\mathrm{p}}^{2}=0.056 ; 500 \mathrm{~ms}: F(1$, $66)=7.17, p=0.009, \eta_{\mathrm{p}}^{2}=0.098 ; 700 \mathrm{~ms}: F(1,66)=3.69, p=0.059, \eta_{\mathrm{p}}^{2}=0.053 ; 900 \mathrm{~ms}: F(1,66)=$ $\left.5.82, p=0.019, \eta_{\mathrm{p}}^{2}=0.081\right)$. These results suggest that visual intentional binding is weaker than auditory intentional binding regardless of subsecond action-outcome temporal discrepancy.

Even though weak in degree, visual intentional binding emerges with small outcome delays as shown in our analysis of Experiment 2 (see 3.2.1) and this was partially consistent with previous findings showing that visual intentional binding is weakened by larger outcome delays (Ruess et al., 2018). Importantly, we observe significant positive intra-individual correlations between intentional binding and agency rating both in auditory and visual domains (Fig. 4), and additionally $t$-test confirm that there was no significant difference in the correlation coefficients between experiments $(t(66)=1.70, p$ $=0.093, d=0.413)$. This suggests that intentional binding may coincide with explicit sense of agency regardless of outcome modality.

\section{General discussion}

This study examined intra-individual correlations between intentional binding (i.e., subjective compression of action-outcome temporal interval caused by agentive situations) and explicit sense of agency over the auditory (Experiment 1) and visual (Experiment 2) action-outcomes, as well as 
inter-individual correlation, which has been examined in previous studies (see next paragraph). In both outcome modalities, outcome delays decreased the explicitly rated sense of agency due to temporal incongruence with outcome timing predictions, as expected (Farrer, Bouchereau, et al., 2008; Sato \& Yasuda, 2005). Intentional binding for auditory outcomes were robustly observed regardless of the outcome delays, and importantly, increasing outcome delays decreased the extent of intentional binding in accordance with previous findings (Haggard et al., 2002). Indeed, we found inter-individual correlations between regression slopes with outcome delay as a predictor for agency rating and intentional binding in the auditory domain. In contrast, although visual outcome did not induce intentional binding in terms of the grand average level (i.e., lack of main effect of the action and baseline tasks, see 3.2.1), post-hoc analysis suggested that the binding was observed only in the small, 100- and 300-ms, delay conditions. It may be that because of the limited emergence of the visual intentional binding, we did not find the abovementioned inter-individual correlation between regression slopes, unlike in the auditory domain. Most importantly we found - for the first time, to our knowledge-intra-individual (i.e., trial-by-trial based) correlations between agency rating and intentional binding in both outcome modalities. This suggests that intentional binding, as an implicit marker of sense of agency, can coincide with the explicit sense of agency over sensory outcomes.

\subsection{Intra- and inter-individual correlation between explicit and implicit sense of agency}

Subjective rating of sense of agency and the degree of intentional binding have been employed as established measures of sense of agency in a vast number of studies (David et al., 2008; Haggard, 2017; Moore \& Obhi, 2012). Our results suggested that these two measures correlate intra-individually (i.e., coincide on a trial by trial basis) and inter-individually (i.e., similar modulation by the amount of action-outcome congruence). Although one might assume that the correlation between these two measures imply that they relate to the same underlying psychological construct, the evidence is mixed with both dissociations as well as associations reported (Braun et al., 2014; Dewey \& Knoblich, 2014; Ebert \& Wegner, 2010; Pyasik et al., 2018; Saito et al., 2015). To account for dissociations, theoretically, different mechanisms may underlie agency judgment and intentional binding, which might be generated at different levels (Hughes, Desantis, \& Waszak, 2013; Synofzik et al., 2008; Synofzik et al., 2013): while agency judgment relies on conceptual and evaluative processes (e.g., "I did it"), intentional binding relies on perception of the action and outcome onset timings and/or action-outcome temporal interval. However, biases in perception of temporal intervals and timing of action and its outcome due to intentional binding, may themselves serve as a cue for explicit sense of agency (Synofzik et al., 2013). Specifically, compressed action-outcome intervals might promote agency judgment based on temporal congruence between one's action and its outcome whereas subjectively reversed action-outcome ordering may cause a denial of one's agency 
(Imaizumi \& Asai, 2015; Timm, Schonwiesner, SanMiguel, \& Schroger, 2014). Given this, it is plausible that degree of intentional binding is (in)directly associated with the degree of perceived sense of agency. Indeed, brain imaging and stimulation studies have suggested that, although frontal and parietal cortices are associated with agency judgment (Farrer, Frey, et al., 2008; Farrer \& Frith, 2002; Hughes, 2018) and intentional binding (Khalighinejad et al., 2016; Moore et al., 2010), respectively, these two aspects of agency may recruit a shared neural mechanism. Explicit sense of agency can also relate to frontal region activities (Nachev et al., 2005; Nachev et al., 2007; Sperduti et al., 2011) and crucially, to front-parietal functional connectivity (Chambon et al., 2013). This suggests that explicit and implicit agency might correlate at neural, and potentially behavioral, levels. This possibility finds support in psychiatric research: people with schizophrenia, who may have a deficit in self-other discriminability (Asai, 2016), show abnormalities of explicit and implicit sense of agency, such as hyper-attribution (Hur et al., 2014) and hypo-attribution (Waters et al., 2012) of one's own action to the self, and enhanced intentional binding (Haggard et al., 2003; Voss et al., 2010) relative to healthy controls.

It has also been suggested that voluntary action and/or sense of agency may themselves modulate time perception. When individuals voluntarily move their body (parts), potentially entailing sense of agency, their subjective time is flexibly modulated based on the phases of movement (i.e., motor preparation and execution). During the period of preparation of ballistic manual movement, subjective durations of stimuli expand and visual temporal resolution is enhanced (Hagura, Kanai, Orgs, \& Haggard, 2012). During execution of voluntary finger lifting movement, spatially congruent video-hand feedback can be perceived to last longer than incongruent feedback (Press, Berlot, Bird, Ivry, \& Cook, 2014). Indeed, such subjective time distortion induced by bodily movements is limited to self-initiated sensory events (Dewey \& Carr, 2013), and covaries with the degree of perceived sense of agency (Imaizumi \& Asai, 2017). Finally, after the completion of a voluntary action followed by sensory events, fluctuation of tactile temporal processing can be observed as well as intentional binding (Wenke \& Haggard, 2009). These previous findings suggest a link between sense of agency and modulation of subjective time; this notion could be supported by the present findings, suggesting that explicit sense of agency is associated with intentional binding even on a trial-by-trial basis. To speculate, fluctuation of subjective time might be an adaptive function driven by sense of agency for optimal selection and planning of "one's own" actions and subsequent or online processing of sensory consequences. Further studies will be needed to empirically evaluate this suggestion, while examining relationships with other aspects of sensory modulation driven by voluntary movements, such as sensory attenuation, whereby perceptual intensity of, and neural response to, sensory inputs are suppressed when they are self-initiated and predictable (Blakemore, Wolpert, \& Frith, 1998; Kaiser \& 
Schutz-Bosbach, 2018; Weiss, Herwig, \& Schutz-Bosbach, 2011).

In previous studies, intra-individual correlations between explicit sense of agency and intentional binding have not been examined and inter-individual correlations have not been found, perhaps because of methodological limitations (see 1.3) in that they employed different motor tasks and stimuli to measure agency judgment and intentional binding (Braun et al., 2014; Saito et al., 2015), or because of offline reports of these two measures (Dewey \& Knoblich, 2014). Furthermore, other studies did not describe the intra-individual correlation analysis even though their experimental design may have been capable of making comparisons between agency judgment and intentional binding (Ebert \& Wegner, 2010; Hughes, 2018; Pyasik et al., 2018; Wen et al., 2015). On the other hand, our two experiments employed measurement on a trial-by-trial basis both for agency rating and interval estimation between the action and its sensory outcome. This allowed us to examine the intra-individual correlation, consequently showing that explicit sense of agency and intentional binding over auditory and visual outcomes were intra-individually, positively correlated. These findings might also support the validity of intentional binding as an implicit marker of sense of agency.

Care should be taken when we interpret intra-individual correlation between agency rating and intentional binding (i.e., estimated-delay ratio) measured in a single trial, because there may be a potential response bias, where a response to one question may prime a corresponding value in the subsequent response to the other question. If so, the intra-individual correlation should be falsely strengthened. Nevertheless, we claim that such response bias may be unlikely in our experiments, because the estimated-delay ratio per se was not directly reported by participants. When computing the intra-individual correlation coefficient, the value of the interval estimation in each trial was converted to an estimated-delay ratio by referring to baseline values determined for each participant (for details, see 2.1.4). On the other hand, our results showed no effect of the question order on the intra-individual correlation coefficients in both experiments, suggesting at least that it is unlikely that either agency rating or interval estimation has a unidirectional effect on the subsequent response to the other measure. However, our results still cannot rule out a possibility that both measures have this unidirectional effect, leading to falsely strong intra-individual correlation. This limitation of the present study should be addressed in future studies.

Our first experiment showed positive inter-individual correlations between sensitivities to outcome delays (i.e., the regression slope) in agency ratings and intentional binding for auditory outcomes, suggesting that explicit sense of agency and intentional binding are similarly modulated by 
action-outcome temporal congruence. This finding is partially consistent with Ebert and Wegner (2010), although they examined inter-individual correlations between sensitivities to spatial congruence of visual outcomes (for influences of outcome modality, see 4.2). Given that temporal and spatial congruence between sensory feedback predicted by motor commands and the actual feedback is one of the key determinants of sense of agency (Blakemore et al., 2000; Frith et al., 2000; Haggard, 2017), our results may also suggest (at least partially) shared mechanisms underlying explicit sense of agency and intentional binding.

\subsection{Differences between outcome modalities}

Experiment 2 showed that visual intentional binding was weaker, limited to smaller outcome-delay intervals and was insensitive to changes in outcome delay (i.e., regression slopes converging to zero, Fig. 3B) relative to the auditory intentional binding in Experiment 1. Weaker intentional binding in the visual domain is consistent with Ruess et al. (2018) although they showed the degree of visual intentional binding was sensitive to outcome delays. This might be due to the use of a different task (i.e., onset timing judgment in Libet's clock paradigm), and/or a different range of outcome delays (150-650 $\mathrm{ms}$ in five steps). Visual inferiority in self-specific processing has also been found in the sensory attenuation effect induced by voluntary actions and/or outcomes matching with internal predictions, with this effect robustly observed in auditory (Curio, Neuloh, Numminen, Jousmaki, \& Hari, 2000; Martikainen, Kaneko, \& Hari, 2005) and tactile domains (Bays, Wolpert, \& Flanagan, 2005; Blakemore et al., 2000). For example, visual spatial resolution is unlikely to be affected by congruence with prediction (Schwarz, Pfister, Kluge, Weller, \& Kunde, 2018). Moreover, there are divergent findings regarding event-related cortical responses to self-initiated visual outcomes (Hughes \& Waszak, 2011; Mifsud et al., 2016). One plausible account for this is the likelihood of pairing between the motor effector and the modality of the action's outcome (Mifsud et al., 2018). Manual action (e.g., tapping) and vocalization are likely to cause auditory events while eye movement causes visual events (i.e., change of view). This idea has been supported by studies suggesting that sensory attenuation of brain responses is found in the effector-modality parings noted above (Mifsud et al., 2018; Mifsud \& Whitford, 2017). To speculate, intentional binging could also rely on the effectormodality coupling such that specific modalities of the action outcome are predicted by an action (e.g., tone by keypress), and subsequently, temporally bound to the action. To date, all studies on intentional binding, to our knowledge, employed manual action only. Thus, the potential effect of effectormodality coupling on intentional binding as well as agency judgment should be examined in the future studies.

A further account comes from behavioral and neural evidence showing stronger functional coupling 
of the motor system with auditory than with visual systems. Temporal control of bodily movements relies strongly on auditory feedback, given the finding of a robust effect by which continuous manual movements are synchronized more to auditory than to visual rhythmic stimuli (Repp \& Penel, 2004; Repp \& Su, 2013). This auditory dominance can be supported by neural evidence showing that synchronization of manual movements to auditory and visual pacing stimuli recruits distinctive brain networks, and in particular, the synchronization to auditory stimuli strongly activates putamen in basal ganglia (Witt, Laird, \& Meyerand, 2008). Although the tighter audiomotor coupling itself does not assume involvement of voluntariness and sense of agency, and does not necessarily suggest modulation of perceived intervals, it might be a potential basis for temporal binding of auditory events driven by voluntary action, presumably resulting in stronger intentional binding.

Alternatively, our cross-experiment analysis indicated that agency ratings in visual and auditory domains were comparable such that the rating values were largest at the minimum outcome-delay conditions and decreased with increasing delays (see Fig. 2A, 6A). Given one view that explicit sense of agency can rely on postdictive inferential processing of causal association between an action and its outcome (Synofzik et al., 2013; Wegner, 2003), it may be plausible that sensory modality at a lower level does not affect inferential agency judgment at a higher level. Thus, although we claim an association between intentional binding and agency judgment, it can be speculated that they might be dissociated depending on the sensory modality of action outcomes.

\subsection{Conclusions}

Explicit sense of agency and intentional binding, as an implicit marker of sense of agency, coincide within individuals while being modulated by action-outcome temporal (in)congruence. This was the case regardless of the audiovisual outcome modality. This study also suggests a weaker intentional binding for visual outcomes than for auditory outcomes, and a reduced sensitivity to action-outcome congruence, perhaps due to the potential likelihood of pairing between motor effector and outcome modality. Given that there might be different processes underlying explicit sense of agency and intentional binding (e.g., Synofzik et al., 2013), the cognitive and neural underpinnings of the observed behavioral coincidence between these two aspects of sense of agency should be further elucidated.

We employed the interval estimation task for measurement of intentional binding not only to follow the related studies (e.g., Ebert \& Wegner, 2010) but also to avoid potential issues in the Libet's clock task. Engbert et al. (2007) pointed out that divided attention into the clock hand and the action or its outcome may bias the estimation of event timing, and that initiation of an action might be triggered by 
particular clock hand positions, undermining the voluntariness of action and/or the sense of agency. Nevertheless, the clock task would enable an examination of the contributions of predictive and postdictive processes (Moore \& Haggard, 2008) and weighting on action and its outcome (Wolpe, Haggard, Siebner, \& Rowe, 2013), to the relationship between intentional binding and explicit sense of agency. Moreover, given that the interval estimation could show the opposite effect of outcome delay on intentional binding (i.e., longer delay causing stronger intentional binding) (Humphreys \& Buehner, 2009; Wen et al., 2015), contrary to findings from the clock task (Haggard et al., 2002; Ruess et al., 2018), intentional bindings measured by the interval estimation and the clock task might originate from different mechanisms. This should be further investigated in future studies, and importantly, such investigation would be beneficial for the replication of our findings and the deeper understanding of neurocognitive origins of intentional binding and sense of agency. 


\section{Funding}

This work was supported by Grant-in-Aids for JSPS Research Fellow (16J00411) and Young Scientists (B) (17K12701) to SI from the Japan Society for the Promotion of Science who had no involvement in the conduct of the study or manuscript preparation.

\section{Declaration of interest}

None.

\section{Acknowledgements}

We would like to thank Tatsuya Kato for his help during data collection in Experiment 2.

\section{Appendix A}

Table A.1. Descriptive statistics of the sense of agency rating and estimated delay in Experiments 1 and 2. Each cell indicates mean value (SD in parentheses).

\begin{tabular}{|c|c|c|c|c|c|c|}
\hline & & \multicolumn{5}{|c|}{ Outcome delay (ms) } \\
\hline & & 100 & 300 & 500 & 700 & 900 \\
\hline \multirow[t]{8}{*}{ Exp. 1} & Sense of agency rating & 7.90 & 6.76 & 5.46 & 4.48 & 3.43 \\
\hline & & $(1.16)$ & $(1.19)$ & $(1.24)$ & $(1.23)$ & $(1.27)$ \\
\hline & Estimated delay in action task (ms) & 102 & 224 & 351 & 479 & 627 \\
\hline & & $(83)$ & $(117)$ & $(139)$ & $(161)$ & $(202)$ \\
\hline & Estimated delay in baseline task & 186 & 312 & 432 & 579 & 711 \\
\hline & $(\mathrm{ms})$ & $(84)$ & $(128)$ & $(157)$ & $(192)$ & $(219)$ \\
\hline & Estimation ratio (baseline/action) & 2.67 & 1.61 & 1.32 & 1.26 & 1.17 \\
\hline & & $(1.83)$ & $(0.75)$ & $(0.36)$ & $(0.27)$ & $(0.21)$ \\
\hline \multirow[t]{8}{*}{ Exp. 2} & Sense of agency rating & 8.25 & 6.69 & 5.18 & 4.22 & 3.20 \\
\hline & & $(0.63)$ & $(0.87)$ & $(1.18)$ & $(1.24)$ & $(1.14)$ \\
\hline & Estimated delay in action task (ms) & 92 & 214 & 358 & 505 & 665 \\
\hline & & $(59)$ & $(86)$ & $(100)$ & $(150)$ & $(153)$ \\
\hline & Estimated delay in baseline task & 102 & 247 & 377 & 512 & 664 \\
\hline & (ms) & $(58)$ & $(80)$ & $(97)$ & $(134)$ & $(132)$ \\
\hline & Estimation ratio (baseline/action) & 1.57 & 1.30 & 1.10 & 1.10 & 1.04 \\
\hline & & $(1.22)$ & $(0.53)$ & $(0.29)$ & $(0.41)$ & $(0.24)$ \\
\hline
\end{tabular}




\section{References}

Asai, T. (2016). Self is "other", other is "self": poor self-other discriminability explains schizotypal twisted agency judgment. Psychiatry Research, 246, 593-600. doi:10.1016/j.psychres.2016.10.082

Asai, T., Kanayama, N., Imaizumi, S., Koyama, S., \& Kaganoi, S. (2016). Development of Embodied Sense of Self Scale (ESSS): exploring everyday experiences induced by anomalous self-representation. Frontiers in Psychology, 7, 1005. doi:10.3389/fpsyg.2016.01005

Asai, T., \& Tanno, Y. (2007). The relationship between the sense of self-agency and schizotypal personality traits. Journal of Motor Behavior, 39(3), 162-168. doi:10.3200/JMBR.39.3.162-168

Asai, T., \& Tanno, Y. (2008). Highly schizotypal students have a weaker sense of self-agency. $\begin{array}{llll}\text { Psychiatry and } \quad \text { Clinical } & \text { 115-119. }\end{array}$ doi:10.1111/j.1440-1819.2007.01768.x

Asai, T., \& Tanno, Y. (2013). Why must we attribute our own action to ourselves? Auditory hallucination like-experiences as the results both from the explicit self-other attribution and implicit regulation in speech. Psychiatry Research, 207(3), 179-188. doi:10.1016/j.psychres.2012.09.055

Barlas, Z., \& Obhi, S. S. (2013). Freedom, choice, and the sense of agency. Frontiers in Human Neuroscience, 7, 514. doi:10.3389/fnhum.2013.00514

Bays, P. M., Wolpert, D. M., \& Flanagan, J. R. (2005). Perception of the consequences of self-action is temporally tuned and event driven. Current Biology, 15(12), 1125-1128. doi:10.1016/j.cub.2005.05.023

Benjamini, Y., \& Hochberg, Y. (1995). Controlling the false discovery rate: a practical and powerful approach to multiple testing. Journal of the Royal Statistical Society: Series B, 57(1), 289-300. doi:10.2307/2346101

Blakemore, S. J., Wolpert, D., \& Frith, C. (2000). Why can't you tickle yourself? Neuroreport, 11(11), R11-16. doi:10.1097/00001756-200008030-00002

Blakemore, S. J., Wolpert, D. M., \& Frith, C. D. (1998). Central cancellation of self-produced tickle sensation. Nature Neuroscience, 1(7), 635-640. doi:10.1038/2870

Block, R. A., \& Zakay, D. (1997). Prospective and retrospective duration judgments: a meta-analytic review. Psychonomic Bulletin \& Review, 4(2), 184-197. doi:10.3758/BF03209393

Braun, N., Thorne, J. D., Hildebrandt, H., \& Debener, S. (2014). Interplay of agency and ownership: the intentional binding and rubber hand illusion paradigm combined. PLoS One, 9(11), e111967. doi:10.1371/journal.pone.0111967

Caspar, E. A., Christensen, J. F., Cleeremans, A., \& Haggard, P. (2016). Coercion changes the sense of 
agency in the human brain. Current Biology, 26(5), 585-592. doi:10.1016/j.cub.2015.12.067

Caspar, E. A., Cleeremans, A., \& Haggard, P. (2015). The relationship between human agency and embodiment. Consciousness and Cognition, 33, 226-236. doi:10.1016/j.concog.2015.01.007

Chambon, V., \& Haggard, P. (2012). Sense of control depends on fluency of action selection, not motor performance. Cognition, 125(3), 441-451. doi:10.1016/j.cognition.2012.07.011

Chambon, V., Wenke, D., Fleming, S. M., Prinz, W., \& Haggard, P. (2013). An online neural substrate for sense of agency. Cerebral Cortex, 23(5), 1031-1037. doi:10.1093/cercor/bhs059

Cohen, J. (1988). Statistical Power Analysis for the Behavioral Sciences (2nd ed.). Mahwah, New Jersey: Lawrence Erlbaum Associates.

Curio, G., Neuloh, G., Numminen, J., Jousmaki, V., \& Hari, R. (2000). Speaking modifies voice-evoked activity in the human auditory cortex. Human Brain Mapping, 9(4), 183-191. doi:10.1002/(SICI)1097-0193(200004)9:4<183::AID-HBM1>3.0.CO;2-Z

David, N., Newen, A., \& Vogeley, K. (2008). The "sense of agency" and its underlying cognitive and neural mechanisms. Consciousness and Cognition, 17(2), 523-534. doi:10.1016/j.concog.2008.03.004

Dewey, J. A., \& Carr, T. H. (2013). Predictable and self-initiated visual motion is judged to be slower than computer generated motion. Consciousness and Cognition, 22(3), 987-995. doi:10.1016/j.concog.2013.06.007

Dewey, J. A., \& Knoblich, G. (2014). Do implicit and explicit measures of the sense of agency measure the same thing? PLoS One, 9(10), e110118. doi:10.1371/journal.pone.0110118

Ebert, J. P., \& Wegner, D. M. (2010). Time warp: authorship shapes the perceived timing of actions and events. Consciousness and Cognition, 19(1), 481-489. doi:10.1016/j.concog.2009.10.002

Engbert, K., Wohlschlager, A., \& Haggard, P. (2008). Who is causing what? The sense of agency is relational and efferent-triggered. Cognition, 107(2), 693-704. doi:10.1016/j.cognition.2007.07.021

Engbert, K., Wohlschlager, A., Thomas, R., \& Haggard, P. (2007). Agency, subjective time, and other minds. Journal of Experimental Psychology: Human Perception and Performance, 33(6), 1261-1268. doi:10.1037/0096-1523.33.6.1261

Farrer, C., Bouchereau, M., Jeannerod, M., \& Franck, N. (2008). Effect of distorted visual feedback on the sense of agency. Behavioural Neurology, 19(1-2), 53-57. doi:10.1155/2008/425267

Farrer, C., Frey, S. H., Van Horn, J. D., Tunik, E., Turk, D., Inati, S., \& Grafton, S. T. (2008). The angular gyrus computes action awareness representations. Cerebral Cortex, 18(2), 254-261. doi:10.1093/cercor/bhm050

Farrer, C., \& Frith, C. D. (2002). Experiencing oneself vs another person as being the cause of an action: the neural correlates of the experience of agency. Neuroimage, 15(3), 596-603. 
doi:10.1006/nimg.2001.1009

Farrer, C., Valentin, G., \& Hupe, J. M. (2013). The time windows of the sense of agency. Consciousness and Cognition, 22(4), 1431-1441. doi:10.1016/j.concog.2013.09.010

Faul, F., Erdfelder, E., Lang, A. G., \& Buchner, A. (2007). G*Power 3: a flexible statistical power analysis program for the social, behavioral, and biomedical sciences. Behavior Research Methods, 39(2), 175-191. doi:10.3758/BF03193146

Fazio, R. H., \& Olson, M. A. (2003). Implicit measures in social cognition research: their meaning and use. Annual Review of Psychology, 54(1), 297-327. doi:10.1146/annurev.psych.54.101601.145225

Fereday, R., \& Buehner, M. J. (2017). Temporal binding and internal clocks: no evidence for general pacemaker slowing. Journal of Experimental Psychology: Human Perception and Performance, 43(5), 971-985. doi:10.1037/xhp0000370

Franck, N., Farrer, C., Georgieff, N., Marie-Cardine, M., Dalery, J., d'Amato, T., \& Jeannerod, M. (2001). Defective recognition of one's own actions in patients with schizophrenia. American Journal of Psychiatry, 158(3), 454-459. doi:10.1176/appi.ajp.158.3.454

Frith, C. D., Blakemore, S. J., \& Wolpert, D. M. (2000). Abnormalities in the awareness and control of action. Philosophical Transactions of the Royal Society B: Biological Sciences, 355(1404), 1771-1788. doi:10.1098/rstb.2000.0734

Gallagher, S. (2000). Philosophical conceptions of the self: implications for cognitive science. Trends in Cognitive Sciences, 4(1), 14-21. doi:10.1016/S1364-6613(99)01417-5

Haggard, P. (2017). Sense of agency in the human brain. Nature Reviews: Neuroscience, 18(4), 196-207. doi:10.1038/nrn.2017.14

Haggard, P., Clark, S., \& Kalogeras, J. (2002). Voluntary action and conscious awareness. Nature Neuroscience, 5(4), 382-385. doi:10.1038/nn827

Haggard, P., Martin, F., Taylor-Clarke, M., Jeannerod, M., \& Franck, N. (2003). Awareness of action in schizophrenia. Neuroreport, 14(7), 1081-1085. doi:10.1097/01.wnr.0000073684.00308.c0

Hagura, N., Kanai, R., Orgs, G., \& Haggard, P. (2012). Ready steady slow: action preparation slows the subjective passage of time. Proceedings: Biological Sciences, 279(1746), 4399-4406. doi:10.1098/rspb.2012.1339

Hornstein, A. D., \& Rotter, G. S. (1969). Research methodology in temporal perception. Journal of Experimental Psychology, 79(3), 561-564. doi:10.1037/h0026870

Hughes, G. (2018). The role of the temporoparietal junction in implicit and explicit sense of agency. Neuropsychologia, 113, 1-5. doi:10.1016/j.neuropsychologia.2018.03.020

Hughes, G., Desantis, A., \& Waszak, F. (2013). Mechanisms of intentional binding and sensory attenuation: the role of temporal prediction, temporal control, identity prediction, and motor 
prediction. Psychological Bulletin, 139(1), 133-151. doi:10.1037/a0028566

Hughes, G., \& Waszak, F. (2011). ERP correlates of action effect prediction and visual sensory attenuation in voluntary action. Neuroimage, 56(3), 1632-1640. doi:10.1016/j.neuroimage.2011.02.057

Humphreys, G. R., \& Buehner, M. J. (2009). Magnitude estimation reveals temporal binding at super-second intervals. Journal of Experimental Psychology: Human Perception and Performance, 35(5), 1542-1549. doi:10.1037/a0014492

Hur, J. W., Kwon, J. S., Lee, T. Y., \& Park, S. (2014). The crisis of minimal self-awareness in schizophrenia: a meta-analytic review. Schizophrenia Research, 152(1), 58-64. doi:10.1016/j.schres.2013.08.042

Imaizumi, S., \& Asai, T. (2015). Dissociation of agency and body ownership following visuomotor temporal recalibration. Frontiers in Integrative Neuroscience, 9, 35. doi:10.3389/fnint.2015.00035

Imaizumi, S., \& Asai, T. (2017). My action lasts longer: potential link between subjective time and agency during voluntary action. Consciousness and Cognition, 51, 243-257. doi:10.1016/j.concog.2017.04.006

Kaiser, J., \& Schutz-Bosbach, S. (2018). Sensory attenuation of self-produced signals does not rely on self-specific motor predictions. European Journal of Neuroscience, 47(11), 1303-1310. doi:10.1111/ejn.13931

Kalckert, A., \& Ehrsson, H. H. (2012). Moving a rubber hand that feels like your own: a dissociation of ownership and agency. Frontiers in Human Neuroscience, 6, 40. doi:10.3389/fnhum.2012.00040

Kanayama, N., Asai, T., Nakao, T., Makita, K., Kozuma, R., Uyama, T., . . Yamawaki, S. (2017). Subjectivity of the anomalous sense of self is represented in gray matter volume in the brain. Frontiers in Human Neuroscience, 11, 232. doi:10.3389/fnhum.2017.00232

Khalighinejad, N., Di Costa, S., \& Haggard, P. (2016). Endogenous action selection processes in dorsolateral prefrontal cortex contribute to sense of agency: a meta-analysis of tDCS studies of 'intentional binding'. Brain Stimulation, 9(3), 372-379. doi:10.1016/j.brs.2016.01.005

Libet, B., Gleason, C. A., Wright, E. W., \& Pearl, D. K. (1983). Time of conscious intention to act in relation to onset of cerebral activity (readiness-potential): the unconscious initiation of a freely voluntary act. Brain, 106(3), 623-642. doi:10.1093/brain/106.3.623

Martikainen, M. H., Kaneko, K., \& Hari, R. (2005). Suppressed responses to self-triggered sounds in the human auditory cortex. Cerebral Cortex, 15(3), 299-302. doi:10.1093/cercor/bhh131

Merchant, H., \& Yarrow, K. (2016). How the motor system both encodes and influences our sense of time. Current Opinion in Behavioral Sciences, 8, 22-27. doi:10.1016/j.cobeha.2016.01.006 
Mifsud, N. G., Beesley, T., Watson, T. L., Elijah, R. B., Sharp, T. S., \& Whitford, T. J. (2018). Attenuation of visual evoked responses to hand and saccade-initiated flashes. Cognition, 179, 14-22. doi:10.1016/j.cognition.2018.06.005

Mifsud, N. G., Oestreich, L. K., Jack, B. N., Ford, J. M., Roach, B. J., Mathalon, D. H., \& Whitford, T. J. (2016). Self-initiated actions result in suppressed auditory but amplified visual evoked components in healthy participants. Psychophysiology, 53(5), 723-732. doi:10.1111/psyp. 12605

Mifsud, N. G., \& Whitford, T. J. (2017). Sensory attenuation of self-initiated sounds maps onto habitual associations between motor action and sound. Neuropsychologia, 103, 38-43. doi:10.1016/j.neuropsychologia.2017.07.019

Molenaar, P. C. M., \& Campbell, C. G. (2009). The new person-specific paradigm in psychology. Current Directions in Psychological Science, 18(2), 112-117. doi:10.1111/j.1467-8721.2009.01619.x

Moore, J. W., \& Haggard, P. (2008). Awareness of action: inference and prediction. Consciousness and Cognition, 17(1), 136-144. doi:10.1016/j.concog.2006.12.004

Moore, J. W., \& Obhi, S. S. (2012). Intentional binding and the sense of agency: a review. Consciousness and Cognition, 21(1), 546-561. doi:10.1016/j.concog.2011.12.002

Moore, J. W., Ruge, D., Wenke, D., Rothwell, J., \& Haggard, P. (2010). Disrupting the experience of control in the human brain: pre-supplementary motor area contributes to the sense of agency. Proceedings: Biological Sciences, 277(1693), 2503-2509. doi:10.1098/rspb.2010.0404

Moore, J. W., Turner, D. C., Corlett, P. R., Arana, F. S., Morgan, H. L., Absalom, A. R., . . Fletcher, P. C. (2011). Ketamine administration in healthy volunteers reproduces aberrant agency experiences associated with schizophrenia. Cognitive Neuropsychiatry, 16(4), 364-381. doi:10.1080/13546805.2010.546074

Nachev, P., Rees, G., Parton, A., Kennard, C., \& Husain, M. (2005). Volition and conflict in human medial frontal cortex. Current Biology, 15(2), 122-128. doi:10.1016/j.cub.2005.01.006

Nachev, P., Wydell, H., O'Neill, K., Husain, M., \& Kennard, C. (2007). The role of the pre-supplementary motor area in the control of action. Neuroimage, 36 Suppl 2, T155-163. doi:10.1016/j.neuroimage.2007.03.034

Obhi, S. S., \& Hall, P. (2011). Sense of agency and intentional binding in joint action. Experimental Brain Research, 211(3-4), 655-662. doi:10.1007/s00221-011-2675-2

Peng, R. D. (2008). simpleboot: simple bootstrap routines. $R$ package version, 1.1-3. Retrieved from https://cran.r-project.org/web/packages/simpleboot/

Press, C., Berlot, E., Bird, G., Ivry, R., \& Cook, R. (2014). Moving time: the influence of action on duration perception. Journal of Experimental Psychology: General, 143(5), 1787-1793. 
doi:10.1037/a0037650

Pyasik, M., Burin, D., \& Pia, L. (2018). On the relation between body ownership and sense of agency: a link at the level of sensory-related signals. Acta Psychologica, 185, 219-228. doi:10.1016/j.actpsy.2018.03.001

R Core Team. (2018). R: a language and environment for statistical computing. Retrieved from https://www.R-project.org/

Repp, B. H., \& Penel, A. (2004). Rhythmic movement is attracted more strongly to auditory than to visual rhythms. Psychological Research, 68(4), 252-270. doi:10.1007/s00426-003-0143-8

Repp, B. H., \& Su, Y. H. (2013). Sensorimotor synchronization: a review of recent research (2006-2012). Psychonomic Bulletin \& Review, 20(3), 403-452. doi:10.3758/s13423-012-0371-2

Ruess, M., Thomaschke, R., \& Kiesel, A. (2018). Intentional binding of visual effects. Attention, Perception, \& Psychophysics, 80(3), 713-722. doi:10.3758/s13414-017-1479-2

Saito, N., Takahata, K., Murai, T., \& Takahashi, H. (2015). Discrepancy between explicit judgement of agency and implicit feeling of agency: implications for sense of agency and its disorders. Consciousness and Cognition, 37, 1-7. doi:10.1016/j.concog.2015.07.011

Sato, A., \& Yasuda, A. (2005). Illusion of sense of self-agency: discrepancy between the predicted and actual sensory consequences of actions modulates the sense of self-agency, but not the sense of self-ownership. Cognition, 94(3), 241-255. doi:10.1016/j.cognition.2004.04.003

Schwarz, K. A., Pfister, R., Kluge, M., Weller, L., \& Kunde, W. (2018). Do we see it or not? Sensory attenuation in the visual domain. Journal of Experimental Psychology: General, 147(3), 418-430. doi:10.1037/xge0000353

Sperduti, M., Delaveau, P., Fossati, P., \& Nadel, J. (2011). Different brain structures related to selfand external-agency attribution: a brief review and meta-analysis. Brain Structure and Function, 216(2), 151-157. doi:10.1007/s00429-010-0298-1

Synofzik, M., Vosgerau, G., \& Newen, A. (2008). Beyond the comparator model: a multifactorial two-step account of agency. Consciousness and Cognition, 17(1), 219-239. doi:10.1016/j.concog.2007.03.010

Synofzik, M., Vosgerau, G., \& Voss, M. (2013). The experience of agency: an interplay between prediction and postdiction. Frontiers in Psychology, 4, 127. doi:10.3389/fpsyg.2013.00127

Timm, J., Schonwiesner, M., SanMiguel, I., \& Schroger, E. (2014). Sensation of agency and perception of temporal order. Consciousness and Cognition, 23, 42-52. doi:10.1016/j.concog.2013.11.002

Voss, M., Moore, J., Hauser, M., Gallinat, J., Heinz, A., \& Haggard, P. (2010). Altered awareness of action in schizophrenia: a specific deficit in predicting action consequences. Brain, 133(10), 
3104-3112. doi:10.1093/brain/awq152

Waters, F., Woodward, T., Allen, P., Aleman, A., \& Sommer, I. (2012). Self-recognition deficits in schizophrenia patients with auditory hallucinations: a meta-analysis of the literature. Schizophrenia Bulletin, 38(4), 741-750. doi:10.1093/schbul/sbq144

Wegner, D. M. (2003). The mind's best trick: how we experience conscious will. Trends in Cognitive Sciences, 7(2), 65-69. doi:10.1016/S1364-6613(03)00002-0

Weiss, C., Herwig, A., \& Schutz-Bosbach, S. (2011). The self in action effects: selective attenuation of self-generated sounds. Cognition, 121(2), 207-218. doi:10.1016/j.cognition.2011.06.011

Wen, W., Yamashita, A., \& Asama, H. (2015). The influence of action-outcome delay and arousal on sense of agency and the intentional binding effect. Consciousness and Cognition, 36, 87-95. doi:10.1016/j.concog.2015.06.004

Wenke, D., \& Haggard, P. (2009). How voluntary actions modulate time perception. Experimental Brain Research, 196(3), 311-318. doi:10.1007/s00221-009-1848-8

Wickham, H. (2009). ggplot2: Elegant Graphics for Data Analysis. New York: Springer-Verlag

Witt, S. T., Laird, A. R., \& Meyerand, M. E. (2008). Functional neuroimaging correlates of finger-tapping task variations: an ALE meta-analysis. Neuroimage, 42(1), 343-356. doi:10.1016/j.neuroimage.2008.04.025

Wolpe, N., Haggard, P., Siebner, H. R., \& Rowe, J. B. (2013). Cue integration and the perception of action in intentional binding. Experimental Brain Research, 229(3), 467-474. doi:10.1007/s00221-013-3419-2

Wolpert, D. M., Ghahramani, Z., \& Jordan, M. I. (1995). An internal model for sensorimotor integration. Science, 269(5232), 1880-1882. doi:10.1126/science.7569931

Yoshie, M., \& Haggard, P. (2013). Negative emotional outcomes attenuate sense of agency over voluntary actions. Current Biology, 23(20), 2028-2032. doi:10.1016/j.cub.2013.08.034 